\title{
EQUIVALENCIA DÉBIL Y FUERTE DE LA CONSTITUCIÓN Y LA DEPENDENCIA
}

\author{
Víctor Manuel Longa Martínez \\ (Universidad de Santiago de Compostela)
}

\section{RESUMEN}

In this article I argue against Matthews (1981) claim about the impossibility of deriving dependencies from constitution. Based on his claim, Matthews defends that there is no strong equivalence between the corresponding theoretical frames.

Contrary to that opinion, I start defending the evident connection between weak and strong equivalencies, and weak and strong generative capacities, and I point out the error that drives Matthews to his wrong hypothesis: he correctly defines the notions of weak and strong equivalence, but he applies the latter imperfectly. If strong equivalence consists not only in the generation of sequences, but also in the attribution of an structural description to each sequence, such descriptions give a knowledge of dependencial properties (at least, the most important ones) mechanically, with the only exception of the descriptions referred to sentences.

Summing up, I do not explicitly affirm the existence of a strong equivalence between constitutional and derivational frameworks, but I demonstrate that in order to deny such an equivalence arguments other than those of Matthews' are required.

\section{Introducción}

Las relaciones de constitución y dependencia han constituido y constituyen, de modo independiente o bien combinado, un tema recurrente en Lingüística y en sintaxis, desde diversos puntos de vista y aproximaciones, tanto puramente teóricas como ligadas a aspectos más concretos. En realidad, ninguna teoría sintáctica podría dejar de referirse a ambas nociones explícita o implícitamente, pues gozan de un peso crucial en el estudio de la estructuración de las secuencias.

En este trabajo se incide específicamente sobre la interconexión existente entre ambas; en concreto, se intentará rebatir a Matthews (1981), demostrando mediante los mismos mecanismos por él utilizados que, contrariamente a lo que afirma, es posible extraer información dependencial de la constitución en 
muchos casos cuando ésta se presenta como relación primitiva en un modelo. Para ello, se mostrará el error que comete este autor cuando sitúa su afirmación en el marco de la equivalencia fuerte entre ambos modelos. En otras palabras, se argüirá a favor de la presencia implícita de la dependencia en la constitución en bastantes ocasiones.

Debido al carácter antes señalado de la constitución y la dependencia como temas recurrentes, no se efectuará una presentación de ambas relaciones (para ello, cfr. especialmente la excelente exposición de Matthews, 1981: 73-84). Simplemente, mencionaré que la constitución se conforma como una relación de cada parte con respecto al conjunto, mientras que la dependencia muestra la relación entre las partes del conjunto, tal como es expresado por Mathews (1981) de modo velado y por Rojo \& Jiménez Juliá (1989: 39 y ss.) explícitamente, entre otros.

Así, el presente trabajo se estructura del siguiente modo: tras scñalar varias asunciones importantes para la argumentación posterior, se expondrán las nociones de capacidad generativa débil y fuerte, relacionándolas con las de adecuación observacional y descriptiva, respectivamente. Aunque estas nociones pertenecen al dominio de la gramática generativa, Matthews las utiliza al indagar en la relación entre constitución y dependencia, por medio de la equivalencia débil y fuerte entre ambos modelos, como se verá. Tras ello, se argumentará contra la afirmación de la imposibilidad de derivar aspectos dependenciales a partir de los constitutivos, señalando una clara contradicción presente en Matthews (1981) relacionada con la equivalencia fuerte. Finalmente, se expondrán las conclusiones más relevantes de este trabajo.

\section{Algunas asunciones básicas}

Antes de comenzar a desarrollar el verdadero objetivo del presente artículo, es necesario señalar la adopción de algunas ideas, íntimamente relacionadas con aquél, y por tanto, que incidirán sobre tal objetivo central.

En primer lugar, las únicas relaciones relevantes sintácticamente para intentar explicar la estructuración de las unidades son la constitución y la dependencia; son, pues, estos dos tipos de relaciones los únicos primitivos en este sentido. Los otros tipos de relaciones sintácticas (cfr. Rojo \& Jiménez Juliá, 1989: cap. 2) son derivables de las dos señaladas de uno u otro modo. No entraré en la consideración de las relaciones funcionales, pues no son en absoluto relevantes para la discusión que plantearé1. En cuanto a las relaciones secuenciales, son derivables de las dependenciales o de las constitutivas, pues cualquier

En todo caso, en Rojo \& Juliá (1989:60) se defiende el carácter mixto de los modelos funcionales, de modo que «participan de alguna de las características propias de los otros dos» (se refieren mediante la última expresión a los modelos constitutivos y a los dependenciales). 
estudio secuencial presupone una determinada conformación de las secuencias, y tal conformación sólo se puede obtener por una de las dos vías señaladas (o por su combinación). Así pues, las relaciones secuenciales se establecen en un momento posterior, no tan 'abstracto' como el relevante a la constitución o a la dependencia ${ }^{2}$. Si tomamos un marco sintáctico específico, como el Modelo Clásico de la gramática Generativa (cfr. Chomsky, 1965), se puede apreciar esto bien, pues la secuencialidad se obtiene a partir de la constitución, relación primitiva en ese modelo; de esta manera, el orden viene ya dado, aunque la secuencialidad puede ser modificada mediante las operaciones transformatorias. Lo mismo se puede señalar en esencia en lo tocante a los aspectos que conforman las relaciones conectivas ${ }^{3}$.

Por ello, es correcta la restricción de Matthews a los dos tipos de relaciones mencionados, constitución y dependencia; sólo ellas dos pueden conformar, o «armar», las secuencias.

Al mismo tiempo, no es posible que la constitución y la dependencia pucdan constituirse en relaciones primitivas a la vez en un mismo marco; nunca ambas cstarán estrictamente en el mismo nivel de jerarquización. Este aspecto es igualmente aludido, aunque de modo implícito, por Matthews.

Sin embargo, una asunción básica de este trabajo, esta vez no compartida por el mencionado autor, consiste en la no existencia de modelos absolutamente puros en sintaxis, y en este aspecto se sitúa la génesis del error de Matthews que será rebatido. Por esta razón, presentaciones como las del propio Matthews (1981), o la de Hudson (1980) son en buena medida artificiosas, porque en ambos casos se presentan la constitución y la dependencia como relaciones puras, y absolutamente independientes una de la otra. Quizás se deban reconocer aspectos metodológicos de presentación en la adopción de esta postura, con vistas a que el carácter expositivo se conforme como más diáfano. De este modo, pudicra ser que esta postura radical ni siquiera fuera asumida por autores como los citados, pero, sea así o no, en el caso de Matthews lo relevante consiste en que por medio de ella afirma que a partir de la constitución no es posible extraer ningún aspecto dependencial, en base al examen de la equivalencia fucrte entre ambas. Sin embargo, este examen es errado. Sea cual sea la relación

2 Esta misma postura es asumida por Matthews (1981: 83) cuando señala que la constitución y la dependencia deben estudiarse independientemente del orden de palabras, de la realización, esto es, de las relaciones secuenciales.

Debe señalarse, para evitar confusiones, que la utilización del término 'relación conectiva' es diferente a la que se puede encontrar en Rojo \& Jiménez Juliá (1989: 44 y ss.). Para ellos las relaciones conectivas tienen un sentido muy similar al de dependencia, incidiendo en los aspectos sintácticos de ésta. Sin embargo, utilizo aquí el término para especificar las relaciones establecidas entre elementos unidos por algún tipo de dependencia referencial, como los reflejados en el ligamiento, o en la conexión entre las huellas y las categorías de las que dependen (por medio de indices, etc). 
primitiva de las dos, tal carácter primitivo no constituye una barrera para conocer de modo derivado aspectos referidos a la otra relación mediante el único recurso de los mecanismos ofrecidos por el modelo.

Recapitulando lo señalado en este apartado, en este trabajo se han asumido las siguientes concepciones, muy relevantes para el mismo, en relación a la constitución y a la dependencia:

A. Sólo el enfoque constitutivo o el dependencial puede conformar las secuencias.

B. Esto significa que, frente a los demás tipos de relaciones, las dos mencionadas son las únicas que pueden gozar del carácter de relaciones primitivas. Las otras, si aparecen, lo harán como derivadas, y se situarán en el plano de la materialización de los resultados obtenidos por la constitución y/o la dependencia; éstas, sin embargo, son previas, conformadas en un momento más abstracto.

C. Constitución y dependencia no pueden ser al tiempo relaciones primitivas. Una de ellas deberá primar necesariamente, pero

D. no de modo que la otra (o cualquiera de las otras derivadas) no pueda ser conocida, al menos en ciertos aspectos; en otras palabras, no existen modelos constitutivos o dependenciales totalmente puros, pues, por ejemplo, una gramática que cuente con la constitución como primitivo posibilitará, gracias a los propios mecanismos constitutivos, la transparencia de relaciones dependenciales (o secuenciales, etc), y lo hará de modo mecánico, sin que la intuición cuente.

\section{Adecuaciones gramaticales y capacidades generativas}

Debido a la utilización que efectúa Matthews de las nociones «equivalencia débil» y «equivalencia fuerte», expondré los conceptos en los que ambas se asientan, que como veremos, son específicamente generativos: capacidad generativa débil y capacidad generativa fuerte, junto a los íntimamente ligados de adecuación observacional y descriptiva.

Imaginemos que mediante un sistema de reglas, que denomino $\mathrm{Sx}$, ha sido conformada una gramática generativa $\mathrm{Gx}$ de una lengua-I(nteriorizada) L dada. Tal gramática, por el mero hecho de haber sido formulada, no tiene por qué ser acertada o adecuada; en términos de Chomsky (1957: 69), la adecuación de la gramática Gx debe ser justificada ${ }^{4}$.

El término «adecuación», en sus diversos grados, es fundamental en la perspectiva descrita, porque será el que permita establecer la pertinencia de la gramática Gx propuesta para dar cuenta de la competencia de la lengua-I L;

4 Por supuesto, tal gramática debe ser evaluada al tiempo en relación con la teoría lingüística que la ha posibilitado, y de este modo, la propia teoría lingüística es a la vez evaluada. Sin embargo, en este trabajo la noción de «teoría lingüística» no es relevante, y por esta razón no se harán alusiones a la misma ni en este apartado ni en el trabajo en general. 
será, en resumidas cuentas, el que permita justificar la gramática en cuestión.

De modo implícito en Chomsky (1957: cap. 6), y ya abiertamente en trabajos como Chomsky (1964:29, y esp., 31 y ss.) y (1965: 19 y ss.) son formulados tres grados o niveles de adecuación de las gramáticas ( $\mathrm{y}$, por extensión, de las teorías lingüísticas que las posibilitan): observacional, descriptivo y explicativo. Ya que este último no tiene relevancia en la discusión que planteo (cfr. n. 4), me centraré en los dos primeros:

1. Adecuación observacional: es el nivel jerárquicamente más bajo de los tres, y básicamente se puede definir como la enumeración taxonómica de un conjunto de secuencias. Éstas pueden ser las de un corpus, bien como conjunto limitado y bien conformado de secuencias (López Morales, 1974: 51), o como conjunto de los «datos primarios» accesibles al niño en el proceso de adquisición del lenguaje (Smith \& Wilson, 1979: 213). Sin embargo, el sentido más interesante de este primer nivel consiste en la posibilidad de que una gramática genere todas las secuencias gramaticales de la lengua, bien la del niño, bien (sobre todo) la del adulto. Por ello, este nivel puede referirse a la competencia, a la posibilidad de generar por medio de los mecanismos con que cuenta la gramática cualquier secuencia posible en esa lengua.

2. Adecuación descriptiva: asumamos que la gramática específica Gx dispone de adecuación observacional en relación a la lengua $L$, esto es, que dispone de capacidad para generar todas y cualquiera de las secuencias gramaticales de L, y sólo ellas. Sin embargo, este requisito, aunque básico, no es suficiente, y por ello debe ser complementado por otro que, así, se sitúa por encima del anterior. Este requisito es precisamente el segundo nivel mencionado, defïnido por Chomsky (1965: 25) con estas palabras:

A una gramática se la puede considerar como una teoría de la lengua, y es descriptivamente adecuada en la medida en que se describe correctamente la competencia intrínseca del hablante nativo idealizado. 5

Aunque ambos niveles se refieren a la misma realidad mental (o, al menos, el observacional debe referirse a ella para ser algo relevante), lo relevante es que lo hacen de forma muy distinta: mientras el nivel observacional genera, hace explícita, la competencia, el descriptivo trasciende esa generación o explicitación, mediante su descripción. Y ésta es una diferencia muy fuerte, porque la gramática no sólo debe proporcionar una explicitación de la competencia, sino también ofrecer indicaciones sobre su estructura, consiguiendo mediante esta condición, por ejemplo, relacionar secuencias de modos diversos ${ }^{6}$. Tales indicaciones sobre

$s$ Cfr. Chomsky (1965: 30) sobre las tres condiciones específicas asociadas a la obtención del nivel de adecuación descriptiva.

6 Recordemos que esta relación entre pares de secuencias es uno de los objetivos básicos del Modelo Clásico del generativismo, y responde a intentar dar cuenta de la relación intuitiva que los hablantes perciben entre secuencias como una activa y su contraparte pasiva. Tal intuición se 
la estructura son of recidas mediante las «Definiciones Estructurales», y precisamente serán éstas las que posibilitan la expresión de generalizaciones lingüísticamente significativas, que expresen las regularidades existentes en un nivel subyacente entre las diversas lenguas particulares (Chomsky, 1964: 29). Al definir no secuencias sino estructuras se puede agrupar a éstas en aquéllas, consiguiendo las mencionadas generalizaciones relevantes.

Las nociones de capacidad generativa débil y fuerte son muy importantes en esta perspectiva; en palabras de Chomsky (1965: 57),

Dada una teoría descriptiva de la estructura del lenguaje, podemos distinguir su capacidad generativa débil de su capacidad generativa fuerte de la siguiente manera: Digamos que una gramática genera débilmente un conjunto de oraciones y que genera fuertemente un conjunto de descripciones estructurales (...). Supóngase que la teoría lingüística T proporciona la clase de gramáticas $G_{1}, G_{2}, \ldots$, donde $G 1$ genera débilmente la lengua $L_{1}$ y genera fuertemente el sistema de descripciones estructurales $\Sigma$ l. Entonces la clase $\left\{\mathrm{L}_{1}, \mathrm{~L}_{2}, \ldots\right\}$ constituye la capacidad generativa débil de $\mathrm{T}$ y la clase $\left\{\Sigma_{1}, \Sigma_{2}\right.$, ... constituye la capacidad generativa fuerte de $\mathrm{T}$.?

Los niveles adecuativos aludidos, observacional y descriptivo, se pueden relacionar con las capacidades o poderes generativos de una gramática, en este sentido: de igual modo que la adecuación observacional consiste en la generación de secuencias, y la descriptiva en la formulación de definiciones sobre las secuencias, la capacidad generativa débil consistirá en la posibilidad de que los mecanismos de la gramática estén en disposición de generar tales secuencias, mientras que mediante la capacidad generativa fuerte los mecanismos de la gramática deberán of recer descripciones sobre la estructura de las secuencias.

Notemos que la relación entre adecuaciones y capacidades es enormemente íntima, porque si una gramática dispone de capacidad generativa débil, dispondrá a la vez de adecuación observacional, y, análogamente, si dispone de la capacidad generativa fuerte, gozará de adecuación descriptiva. Así, las capacidades son la condición previa para poder atribuir a una gramática generativa adecuación en los dos niveles señalados: una gramática dispondrá de adecuación en sus dos vertientes si antes demuestra tener un poder generativo correcto en cada caso. Por ello, adecuaciones y capacidades hacen referencia a lo mismo en esencia, pero la noción de capacidad incide más bien sobre los mecanismos de la gramática, mientras que mediante la adecuación se traslada esa cuestión al

explica mediante la identidad de estructura profunda para ambas oraciones; el caso inverso lo tenemos en secuencias del tipo «el loro de Leoncio no para de hablar», donde a dos estructuras profundas le corresponde una sola superficial.

7 Recordemos que el nivel de la teoría lingüística no es relevante en el presente trabajo (cfr. n. 4). Por ello, se considerará sólo la capacidad generativa en relación a una gramática, no a la teoría que la permite. 
campo psicológico, evaluando la pertinencia de una gramática en relación a la realidad mental del hablante-oyente.

Como se podrá apreciar, la cuestión de la capacidad generativa, en su doble vertiente, es crucial, porque si el poder generativo débil falla por defecto, no será posible hacer explícita la totalidad de la competencia (o, al menos, tener la posibilidad de hacerlo), mientras que si el fallo es por exceso, será todavía más grave, puesto que en este caso serían generadas más secuencias que las pertenecientes a la competencia (agramaticales, etc). En relación a la capacidad fuerte, un fallo por exceso o por defecto en ella supondría añadir el problema de la inadecuación (o ausencia) de las Definiciones Estructurales a la generación de las secuencias, con lo que algunas secuencias podrían quedar sin una descripción de su estructura, o incluso, se podrían adjudicar estructuras erróneas a las secuencias ${ }^{8}$.

Hasta ahora, me he referido a las capacidades generativas (y a las adecuaciones) en una sola gramática $G$, pero si trasladamos la presentación a dos gramáticas, $\mathrm{G}^{1}$ y $\mathrm{G}^{2}$, propuestas para una sola lengua $\mathrm{L}$, tales dos gramáticas serán débilmente equivalentes si pueden generar (o, por lo menos, reconocer) las mismas secuencias, mientras que serán fuertemente equivalentes si pueden ofrecer la misma descripción de la estructura para cada secuencia. En términos de Weinberg (1988: 482), mientras la cuestión de la equivalencia débil entre las dos gramáticas en relación a las capacidades generativas requiere una respuesta «sí/no», la equivalencia fuerte presupone mucho más que una respuesta de ese tipo.

Tras presentar las nociones aludidas de un modo teórico, me ha interesado especialmente introducirlas cuando debemos dilucidar entre dos gramáticas, pues en este caso ambas se enfrentarán en base a su equivalencia débil y fucrte. En realidad, ésta constituye la base de la discusión a Matthews (1981), que hace referencia a la equivalencia débil y fuerte entre un modelo constitutivo y otro dependencial, como a continuación se mostrará.

8 Obvío en la presente exposición un problema muy grave en Chomsky (1965): la posibilidad de aplicar varias Definiciones Estructurales a una misma secuencia, debido al poder tan enorme del aparato expresivo de la gramática, lo que se traducía en su fortísimo poder descriptivo. Esto es, cn la práctica, existía un fallo por exceso en la formulación de las descripciones sobre la estructura de las secuencias. En este sentido es clásico el debate entre Bach (1962) y Bierwisch (1963) y por otro lado Ross (1967) sobre el orden de palabras en alemán; lo relevante es que en ambos casos se ofreen diferentes asignaciones de estructuras sobre los mismos datos, y la teoría se ve imposibilitada para decidir. Pero esta posibilidad no se restringía al componente sintáctico: Harms (1966) y Chomsky \& Halle (1968) proponen dos componente fonológicos distintos para una gramática del payute del sur, y de nuevo, ambas descripciones son compatibles con los datos, idénticos en ambos casos. Por problemas como el señalado, se hizo necesario restringir de modo muy fuerte los mecanismos de la gramática. 


\section{Equivalencia débil y fuerte entre constitución y dependencia}

Tras presentar en los dos primeros apartados del capítulo la constitución y la dependencia por separado, el objetivo de Matthews en el tercero (págs. 84 y ss.) consiste en comparar ambos enfoques, mediante los parámetros de la equivalencia débil y la equivalencia fuerte, parámetros que están claramente jerarquizados (como lo están las capacidades generativas y las adecuaciones): el segundo, la equivalencia fuerte, es más interesante que el primero, y además, mientras que si obtenemos el primero no llegamos por ello automáticamente al segundo, si logramos cl segundo a la fuerza deberemos haber pasado por el primero.

Con respecto a la equivalencia débil, Matthews (1981: 84) señala que

In general, it can be shown that for any dependency grammar there is a phrase structure grammar which will generate an identical set of sentences; likewise, for any phrase structure grammar (or any phrase structure grammar limited to the form of rule which we have illustrated), the same set of sentences can be generated by a dependency grammar. In that sense the two are said to be weakly equivalent.

En resumen, existirá equivalencia débil entre dos gramáticas si cada una de ellas puede generar el mismo conjunto de secuencias que la otra. Definida de este modo, la equivalencia débil se vincula automáticamente con la capacidad generativa débil, sólo que trasladada al plano de la comparación entre varias gramáticas alternativas, por lo que se puede apreciar que este autor se basa en la noción de capacidad generativa de modo claro ${ }^{10}$. Recordemos que la adecuación observacional consiste en la generación de las secuencias permitidas por la gramática, mientras que la capacidad generativa débil consiste en que los mecanismos de la gramática puedan lograr esa generación. Por ello, si la equivalencia débil consiste para Matthews, tal como él mismo afirma, en generar «an identical set of sentences $»$ ( $\mathrm{sic}$ ), no cabe duda de que la equivalencia débil consiste en la comparación de la capacidad generativa débil entre dos o más gramáticas, en este caso, particularmente, si la constitución puede generar las mismas secuencias que la dependencia y viceversa. Me interesa, en todo caso, dejar bien clara esta identificación, porque el mencionado autor formulará una clara contradicción en el nivel de la equivalencia fuerte.

En la misma página (pág. 84) se pregunta, en relación a la equivalencia débil, lo siguiente:

- Se refiere a reglas de rescritura, del tipo de las postuladas en Chomsky (1965), y en general, en toda la GGT, al menos hasta la consolidación definitiva de la sintaxis X-con-barras.

in Incluso Matthews se refiere en varias ocasiones explícitamente al término 'generación', lo que reafirma esta adopción generativa, realizando esa generación mediante reglas de rescritura (cfr. n. anterior). 
If the models are wcakly equivalent, how do they compare in other and more interesting senses?

Esta cita pone de manifiesto que, al igual que lo que sucede con la adecuación observacional frente a la descriptiva, y a la capacidad generativa débil frente a la fuerte, la equivalencia débil entre dos o más gramáticas no es demasiado relevante; de ahí que Matthews se refiera a «other and more interesting senses». Por ello, intuimos que este sentido más interesante debe referirse a algo equivalente a la capacidad generativa fuerte, y efectivamente, Matthews (1981: 84) introduce la equivalencia fucrte como esa noción más interesante que compara dos gramáticas. Es éste el sentido al que implícitamente se aludía en la cita anterior, como podemos apreciar:

Is there anything we can say in a dependency grammar that we cannot also say, in substance, in a phrase structure grammar? Conversely, is there anything we can say in a phrase structure grammar that we cannot also say, in substance, in a dependency grammar? If both answers are no, the models are strongly equivalent

La identificación entre equivalencia fuerte y capacidad generativa fuerte es en realidad clarísima cuando a continuación Matthews especifica en qué consiste la primera (pág. 84):

For any grammar of either type, there will be a grammar of the other type which not only generates an identical set of sentences, but also describes the construction of each sentence in a way that is effectively identical.

Recordemos de nuevo que la diferencia entre la capacidad generativa débil y la fuerte reside en que mientras la primera genera las secuencias permitidas por la gramática gracias a los mecanismos de ésta, la segunda trasciende este ámbito, pues además agrega a la generación una definición de la estructura de las secuencias. Fijémonos en que si Matthews ha definido la equivalencia débil como generación de las secuencias a secas y la fuerte como descripción de la estructura de las mismas, tal descripción a la fuerza debe entenderse como especificación de la estructura de las secuencias, y no especificación del simple resultado de la generación, esto es, de las secuencias generadas. Así, el término «descripción» alude a algo que trasciende la mera enumeración.

Así pues, asumo el paralelismo estricto entre los dos pares de conceptos mencionados (equivalencias y capacidades). Esto, como se verá en seguida, es muy importante.

Dejemos este aspecto por el momento, y centrémonos en otra afirmación de Matthews; tal como he recogido en una cita suya, la equivalencia fuerte se produce si el enfoque dependencial y el constitutivo pueden ofrecer la misma 
información; si se produce la posibilidad contraria, no existirá tal grado de equivalencia. Esto significa que las descripciones estructurales of recidas por cada uno de los dos enfoques deben especificar, o al menos, dejar ver, las mismas relaciones, aunque tales descripciones no sean idénticas. En otras palabras, si la información constitutiva puede derivarse de la dependencial y viceversa, aunque en cada caso prime una de ellas, obtendremos la misma carga informativa para la secuencia, y existirá equivalencia fuerte. Por el contrario, si de las relaciones constitutivas expresadas como primitivo por la definición de la estructura, nos vemos imposibilitados para conocer las dependenciales y viceversa, no existirá tal grado de equivalencia.

Por supuesto, tal obtención de información a partir de la relación primitiva debe ser automática, mecánica, como el propio Matthews señala. Por descontado, podemos conocer las dependencias a partir de la constitución y viceversa, ya en el nivel de la equivalencia débil, ya en el de la fuerte, mediante nuestras intuiciones, pero lo verdaderamente relevante consistirá en que sea la propia gramática la que permita deducir esos aspectos mediante el único recurso de los procedimientos usados por ella, independientemente de las intuiciones.

Sobre la posibilidad de la existencia de equivalencia fuerte entre constitución y dependencia Matthews (1981: 84) señala que

Let us imagine that the models are strongly equivalent. In that case there must be a procedure by which the constituents of a sentence can be derived from its dependency relations, and another procedure by which the dependencies can be derived from the constituency.

Estas palabras constituyen el punto central de mi argumentación, por cuanto señala que la segunda opción, consistente en derivar la dependencia por medio

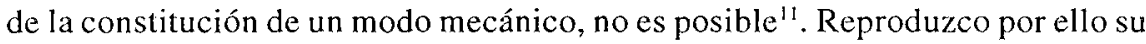
opinión (págs. 84-5) con meticulosidad:

But we can see at once that the second of these procedures ${ }^{12}$ cannot exist. For given a bracketing

$[x \quad[y \quad z]$

there is no way of determining wheter the dependencies are like this:

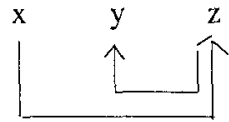

or like this:

" La otra posibilidad, deducir la constitución de la dependencia, no será prácticamente tocada en este trabajo, por no ser especialmente relevante en relación a los objetivos que el trabajo persigue.

:2 El segundo de los procedimientos al que se refiere se puede encontrar en la cita reproducida anteriormente: extraer las dependencias del enfoque constitutivo. 


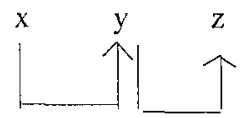

or indeed like this:

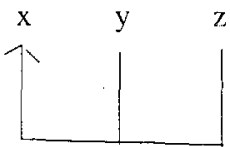

or like this:

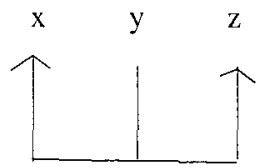

Así pues, Matthews asume que no es posible deducir las dependencias mecánicamente a partir de la constitución, pero ya que sitúa su afirmación en el contexto de la equivalencia fuerte, debemos entenderla, lógicamente, referida a ese nivel en concreto, y no al de la débil. Pero precisamente defender esta opacidad de los aspectos dependenciales a partir de los constitutivos constituye un claro error, desde el momento en que define la equivalencia fuerte como una descripción de la estructura de la secuencia, y no como una especificación de las secuencias en tanto que ristras de elementos léxicos que las conforman, pues es éste el sentido de la equivalencia débil. Además, la estructura que Matthews propone adjudicar a las secuencias consiste en reglas de rescritura, algo que consolida mi argumentación, como ahora se mostrará.

Por ello, si tal afirmación de Matthews sobre la no posibilidad de deducir la dependencia a partir de la constitución se refiriera a la equivalencia débil, básicamente debería ser considerada correcta, pues no existe modo de decidir mecánicamente sobre la dependencia de unos elementos con respecto a otros $\mathrm{en}$ relación a los aspectos constitutivos que conforman cada secuencias (por supuesto, tampoco existiría la posibilidad contraria). Si consideramos una secuencia (no oracional) supuestamente generada por el modelo constitutivo como

[el [niñol]

podemos percibir que a partir de la constitución de la secuencia, expresada por medio de los corchetes, no podemos extraer, sin embargo, ningún aspecto de la dependencia entre los dos elementos integrantes de tal secuencia; no podemos conocer de modo mecánico si el elemento 'el' depende del otro elemento, o si él mismo ejerce dependencia; lo mismo se puede aplicar al constituyente «niño».

Pero el problema de la afirmación mencionada de Matthews consiste en situarla en el nivel de la equivalencia fuerte (recordemos que un momento antes señala que debe sobrepasarse el nivel de la débil); en este caso, la corrección de 
tal hipótesis se anula, porque es relativamente fácil mostrar que a partir de la constitución sí es posible obtener información dependencial, y lo que es más importante, obtenerla de modo mecánico, ciñéndonos exclusivamente a los propios mecanismos de la gramática. Si adjudicamos una estructura a la secuencia anterior, para situarnos en el contexto de la equivalencia fuerte, tenemos lo siguiente:

$\mathrm{FN} \rightarrow$ Det $\mathrm{N}^{13}$

Mediante la regla rescritural formulada se propone una definición de la estructura de la secuencia. En tal regla se explicita el tipo de unidad al que pertenece el conjunto, al tiempo que igualmente se señala cl tipo de unidad al que pertenece cada elemento conformador de la secuencia por separado. Además, se señalan las relaciones constitutivas, como primitivos ${ }^{14}$. Pero a partir del marco constitutivo, frente a lo argumentado por Matthews, sí es posible obtener información dependencial de modo mecánico. Son los propios procedimientos y mecanismos de la gramática los que motivan que en la estructura anterior, repetida aquí

\section{$\mathrm{FN} \rightarrow$ Det $\mathrm{N}$}

se indique la información de que un elemento determinado en cuanto al tipo de unidad, como es el elemento $\mathrm{N}$ («niño») pertenece precisamente al mismo tipo categorial que la totalidad de la estructura, si bien en niveles diferentes, léxico en un caso y frasal en otro. Esta igualdad de tipo categorial, obtenida mecánicamente a partir de los mecanismos de la gramática (esto es, independientemente de toda ayuda de la intuición) no es en modo alguno fruto de la casualidad: es el elemento $\mathrm{N}$ el que conforma a su imagen y semejanza categorial la secuencia en la que se ve involucrado; en otras palabras es este elemento el que da carácter a la frase.

El tipo de unidad provee en este caso el marco para la relación constitutiva, relación primitiva, pero tal relación no es una barrera que impida percibir las dependencias, al menos en los ejemplos en los que están involucradas frases. Por ello, ya que en el ejemplo usado se da la especificación del tipo de unidad además de la especificación de las relaciones constitutivas de la secuencia, ¿se puede sostener que, al menos en este tipo de ejemplos, no se puede deducir la dependencia a partir de la constitución?

13. Notemos que mi argumentación utiliza el mismo tipo de descripciones sobre las estructuras que usa el propio Matthews, por lo que el análisis no tergiversa en modo alguno los procedimientos usados por ese autor.

14 Por ello, asumo que el tipo de unidad por sí mismo no ofrece este tipo de relaciones (ni las dependenciales); las relaciones constitutivas se presentan en un modelo de este tipo cuando insertamos el tipo de unidad en reglas rescriturales. Lo mismo señalan Rojo \& Jiménez Juliá (1989: 58) cuando señalan que «Lo que no cabe en modo alguno es un marco sintáctico en el que las relaciones no sean primitivos teóricos, esto es, donde se llegue a las relaciones a partir de otros conceptos teóricos (por ejemplo, los tipos de unidad)». 
Llevado por la afirmación discutida, y también por la contraria, conforme a la que no es posible obtener información constitutiva a partir de la dependencia, Matthews (1981: 88) propone un modelo mixto que palíe la, a su juicio, inexistencia de equivalencia fuerte; con sus palabras,

In short, there are things we can say in a constituency grammar which we cannot say in a dependency grammar, just as there are other things which we can say in a dependency grammar but not in a constituency grammar. At least some of these things are of descriptive value. As a first step we might therefore attempt to combine both forms of representation. (... $)^{\text {t5 }}$

One rule for noun phrases could likewise be amended to

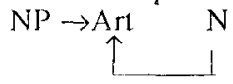

$(\ldots)^{16}$

Si se asume la crítica efectuada a la opinión de Matthews sobre la imposibilidad de derivar las dependencias a partir de la constitución, nos daremos cuenta de que en vez de ayudar a paliar la inexistencia de equivalencia fuerte, el modelo mixto propucsto por Mathews es claramente redundante, pues la información está reduplicada. La flecha que según este autor debe expresar la relación dependencial es claramente redundante, porque tal información aparece ya de modo implícito en la propia regla rescritural. Por ello, no sucede que esté de más la información dependencial, sino la flecha que indica tal relación, porque la dependencia ya aparece en la constitución.

Es a este punto al que quería llegar, pues de lo señalado se puede derivar la existencia implícita (esto es, no primitiva) de información dependencial en la rescrituración, o en el trasvase de ésta al ámbito de las relaciones sintácticas, en la constitución. En otras palabras, la definición estructural propuesta para la secuencia especifica, de modo derivado con respecto a la constitución, aunque igualmente de modo mecánico y claro, las dependencias: si $\mathrm{N}$ aparece en una secuencia $\mathrm{FN}$, será precisamente $\mathrm{N}$ el elemento que conforme tal proyección en la dirección categorial expresada por ese elemento. Por ello, el elemento distinto de $\mathrm{N}$ será a la fuerza un elemento dependiente de él. Naturalmente, a esta visión se lc podría objetar el que sea el tipo de unidad y no la constitución la que, en primera instancia, expresa la identidad categorial (aunque no de proyección) entre $\mathrm{N}$ y FN, por ejemplo. Sin embargo, notemos que es precisamente la constitución la que permite relacionar ambos tipos categoriales, pues mediante ella se señala que $\mathrm{N}$ es una parte de una estructura que, como conjunto, se

is Omito un ejemplo referido a una secuencia oracional; este tipo de estructura será discutido más adelante brevemente.

16 En la pág. 89 vuelve a proponer la combinación de ese modelo 'mixto', en referencia esta vez a la estructura $\mathrm{PP} \rightarrow \mathrm{P}$ NP 
conforma como una FN. Esto es, el tipo de unidad, sin ser insertado en una relación constitutiva, no llegaría para conocer esa relación, pues no existiría nada que pudiera ser relacionado dentro ( $y$ esto es lo relevante) de una estructura concreta.

Tomemos unas cuantas secuencias más, como

(yo) como la carne

en los bosques

difícil de predecir

antes del ocaso

Si extendemos el razonamiento anterior a la consideración de estas secuencias, deberemos proponer una definición de su estructura en cuanto a la in/ existencia de equivalencia fuerte. Tales definiciones pueden ser como las siguientes:

$$
\begin{aligned}
& \mathrm{FV} \rightarrow \mathrm{V} \text { FN } \\
& \mathrm{FP} \mathrm{P} \rightarrow \mathrm{FN} \\
& \mathrm{Fadj} \rightarrow \mathrm{Adj} \text { FP } \\
& \mathrm{Fadv} \rightarrow \mathrm{Adv} \mathrm{FP}^{17}
\end{aligned}
$$

En cualquiera de los integrantes de esta ristra de definiciones estructurales, la proposición del modelo «mixto» de Matthews sería, al igual que en el ejemplo discutido anteriormente, claramente redundante, pues la supuesta explicitación de la relación dependencial por medio de una flecha no añade nada que no pueda ser derivado mecánicamente, constituyendo, de nuevo, una información ya conocida mediante los propios recursos de la gramática.

En esos ejemplos, sucesivamente un elemento $V$ conforma una proyección $\mathrm{FV}$, del mismo modo que los elementos $\mathrm{P}$, Adj y Adv conforman respectivmente una FP, FAdj y FAdv. En todos los casos, de nuevo, un elemento da carácter a la secuencia en la que se integra, de modo que el tipo de unidad resultante no es en absoluto casual. Además, por ello, los elementos distintos del que conforma la secuencia a su imagen categorial se presentan como dominados.

En último extremo, tal elemento central hace alusión a la noción de «núcleo». Por ello, aunque el enfoque sigue siendo constitutivo, el concepto de núcleo está presente implícitamente en él. ${ }^{18}$

17 Aunque en la GGT no se suele trabajar específicamente con Frases Adverbiales (en especial desde el triunfo de la X-con-barras), debido a la consideración de que los Adverbios se pueden reducir a variantes de ciertas preposiciones (cfr. Jackendoff, 1977: 78), como ejemplificación es relevante para los intereses propuestos.

is Este mismo razonamiento, aunque extendido, es el adoptado en Longa (1994) para explicar el aparentemente tan fuerte cambio entre un modelo constitutivo como Chomsky (1965) y otro dependencial como Chomsky (1981). Si se hace explícita la noción de núcleo, podemos obtener el esquema común que ofrece la X-con-barras. Por supuesto, tal esquema unitario no fue posible hasta conseguir reducir la oración a la endocentricidad, lo que se logra en Chomsky (1986). Además, en tal cambio entran en juego más factores, como la pertenencia de la sintaxis X-con-barras a la categoría de regla y principio a la vez. 
Naturalmente, la anterior afirmación sobre la nuclearidad debe ser matizada con atención; si proponemos una definición de la estructura de una secuencia oracional, del tipo

\section{$\mathrm{O} \rightarrow \mathrm{FN} \quad \mathrm{FV}$}

a partir de ella no podremos deducir ningún aspecto dependencial, pues a diferencia de los ejemplos anteriores, no existe un elemento que reduzca el todo resultante al mismo tipo categorial al que ese supuesto elemento pertenece. En todo caso, quizás sería posible deducir, precisamente por la falta de un elemento de ese tipo, que no se establece ningún género de dependencia de un elemento con respecto al otro. Tal imposibilidad de visualizar aspectos dependenciales nos llevaría, en este sentido, a la exocentricidad.

Por otro lado, si consideramos una secuencia algo más compleja que el primer ejemplo aducido, como

el niño rico

y proponemos una definición estructural para la misma, del estilo

FN $\rightarrow$ Det $N$ Adj

estaremos en disposición de deducir información dependencial, aunque no de modo exhaustivo: sí se conocerá mecánicamente la información dependencial más relevante o de primer nivel, la relación existente entre $\mathrm{N}$ y $\mathrm{FN}$, pero no habrá modo de conocer las dependencias de segundo nivel, las establecidas entre los elementos directamente no conformadores de la estructura. Existen varias posibilidades, a las que en general alude Matthews (págs. 84-5): bien $\mathrm{N}$ puede regir a Det y Adj al mismo tiempo, o bien puede existir una dependencia de segundo nivel entre Art y Adj o viceversa.

Sin embargo, creo que los dos aparentes contraejemplos a mi argumentación en realidad no la invalidan. Notemos que no he defendido que existiera una equivalencia fuerte estricta entre ambos enfoques, sino que la crítica se ha referido a que, en opinión de Matthews, nunca existiera la misma. Además, tengamos en cuenta dos consideraciones:

1. Tal información dependencial implícita está presente en la mayor parte de definiciones sobre las estructuras que los mecanismos de la gramática permiten formular, esto es, en la mayor parte de reglas rescriturales, aquéllas en las que un elemento frasal aparece a la izquierda de la flecha. Únicamente la definición estructural de $\mathrm{O}$ se aparta de ese arquetipo. Tengamos en cuenta además que si todas las estructuras como conjuntos dispusieran de una definición estructural tal que se pudiera deducir su carácter a partir del carácter de un elemento específico de la estructura, seguramente no habría modo de sostener la constitución como relación primitiva; la noción de núcleo estaría presente en todas las definiciones estructurales. En este caso, más bien la dependencia se erigiría en relación primitiva, pasando la constitución a relación derivada, pues la endocentricidad constituye claramente un enfoque dependencial (es esto preci- 
samente lo que sucede en la X-con-barras). Por ello, quizás en este caso la discusión planteada no tendría demasiado sentido ${ }^{19}$.

2. Por otro lado, aunque efectivamente en secuencias algo complejas sea imposible determinar de modo mecánico la existencia o la dirección de las dependencias de segundo nivel, no es menos cierto que sí es posible extraer la información dependencial más relevante para la secuencia, la que incide sobre el elemento específico que da carácter al conjunto.

\section{Conclusiones}

En este trabajo se ha intentado argumentar contra la opinión de Mathews (1981) según la cual a partir de un enfoque constitutivo no es posible conocer ningún aspecto dependencial de modo mecánico. Tras explicitar ciertas asunciones, se ha argumentado a favor de la no existencia de modelos absolutamente puros, como muestra la crítica a la creencia de Matthews; en este aspecto reside la génesis del error con respecto a su afirmación anteriormente mencionada.

Matthews sostiene que no existe equivalencia fuerte entre la constitución y la dependencia porque (dejando el otro caso de lado) no es posible deducir ningún aspecto dependencial a partir de la constitución. Pero si define este grado de equivalencia como la descripción de una secuencia en términos de su estructura, y si se hace esa descripción como él la efectúa, mediante reglas de rescritura, está claro que, a diferencia de lo que sucede con la equivalencia débil, donde sólo estamos ante elementos léxicos en los que es imposible decidir mecánicamente, en la fuerte sí es posible conocer de modo mecánico aspectos dependenciales desde la óptica constitutiva. En la mayor parte de tipos de definiciones estructurales (las frases) existe un elemento que da carácter al todo en el que se inserta. En realidad, estamos ante la noción implícita de núcleo. Por todo esto, la tesis de Matthews no se sostiene. Esa misma tesis motiva que este autor proponga un modelo consistente en indicar las dependencias mediante una flecha, pero la información facilitada por la flecha es claramente redundante, porque no se agrega nada a lo ya conocido. Tal flecha, relevante en todo caso para mostrar las dependencias de segundo nivel es innecesaria para conocer la dependencia principal, consistente precisamente en la identidad en cuanto al tipo categorial entre un elemento y el conjunto en el que aparece.

19) Esto me hace pensar que a partir de las dependencias sí es posible obtener la información constitutiva de modo mecánico, con tal de que las dependencias, por supuesto, sean exhaustivas. Al menos dentro de la GGT, el modelo 'Government \& Binding' muestra, frente al Modelo Clásico, que la dependencia es superior (en términos de Hudson (1980), aunque por supuesto, usando diferentes razonamientos) porque (i) mediante la dependencia como primitivo se puede deducir la constitución y (ii) la elevación de la dependencia a primitivo simplifica mucho los mecanismos de la gramática. A esto hace referencia la X-con-barras como principio (no como regla). Desde luego, no me atrevo a generalizar tal afirmación a aquellos modelos no pertenecientes a la GGT. 
Por ello, frente a lo señalado por Matthews, sí es posible conocer mecánicamente aspectos dependenciales (bien los principales, bien todos ellos, dependiendo de la complejidad de la estructura) a partir de la constitución. En resumen, Matthews define bien la equivalencia fuerte, pero cuando la utiliza como argumento para defender que no es posible conocer la dependencia a partir de la constitución, comete el error de ignorar que está trabajando con definiciones sobre las estructuras, y no con sólo secuencias generadas. En todo caso, el objetivo de este trabajo no era afirmar o negar la existencia de equivalencia fuerte entre los dos modelos, sino utilizarla como argumento para mostrar la, a mi juicio, incoherente formulación del mencionado autor. Esto es, puede que no exista equivalencia fuerte entre ambos modelos, pero para demostrar eso no se puede aducir como prueba que la constitución sea una barrera que impida visualizar aspectos dependenciales, porque tal prueba se desmorona en la mayor parte de casos si se analiza con algo de atención.

\section{Referencias bibliográficas}

BACH, E. (1962), "The Order of Elements in a Transformational Grammar of German", Language, 38, 263-269.

BIERWISCH, M. (1963), Grammatik des Deutschen Verbs, Studia Grammatica. CHOMSKY, N. (1957), Syntactic Structures, The Hague, Mouton. Cit. por la trad. esp. de C. Otero, Estructuras sintácticas, Méjico, Siglo XXI, 1974.

CHOMSKY, N. (1964), Current Issues in Linguistic Theory, The Hague, Uitgeverij Mouton \& Co. Cit. por la trad. esp. de G. Ánfora, Problemas actuales en teoría lingüística. Temas teóricos de gramática generativa, Madrid, Siglo XXI, 1978.

CHOMSKY, N. (1965), Aspects of the Theory of Syntax, Cambridge, Mass., The MIT Press. Cit. por la trad. esp. de C. Otero, Aspectos de la Teoría de la Sintaxis, Madrid, Aguilar, 1971.

CHOMSKY, N. (1981), Lectures on Government and Binding, Dordrecht, Foris.

CHOMSKY, N. (1986), Barriers, Cambridge, Mass., The MIT Press. Hay trad. esp. de S. Alcoba y S. Balari, Barreras, Barcelona, Paidós, 1990.

CHOMSKY, N. \& M. HALLE (1968), The sound pattern of English, New York, Harper \& Row. Hay trad. esp. de J.A. Millán y P. Calvo, Principios de fonología generativa, Madrid, Fundamentos, 1979.

HARMS, R.T. (1966), "Stress, voice and lenght in Southern Paiute", International Journal of American Linguistics, 2, 228-235.

HUDSON, R.A. (1980), "Constituency and dependency", Linguistics, 18, 179198. 
JACKENDOFF, R.S. (1977), X `Syntax: A Study of Phrase Structure, Cambridge, Mass., The MIT Press.

LONGA, V.M. (1994), "Relaciones sintácticas en la teoría transformacional: Constitución vs. Dependencia, de ATS a GB", ms., Universidade de Santiago de Compostela.

LÓPEZ MORALES, H. (1974), Introducción a la lingüística generativa, Madrid, Alcalá.

MATTHEWS, P.H. (1981), "Constituency and dependency", en Matthews, P.H., Syntax, Cambridge, Cambridge University Press, 71-95.

ROJO, G. \& T. JIMÉNEZ JULI_Á(1989), Fundamentos del análisis sintáctico funcional, "Lalia", n², Santiago de Compostela, Universidad de Santiago. ROSS, J.R. (1967), "Gapping and the order of constituents", Proceedings of the $X$ International Congress of Linguists.

SMITH, N. \& D. WILSON, Modern Linguistics. The Results of Chomsky 's Revolution, London, Penguin Books. Cit. por la trad. esp. de R. Pochtar, La lingüística moderna. Los resultados de la revolución de Chomsky, Barcelona, Anagrama, 1983.

WEINBERG, A.S. (1988), "Mathematical properties of grammars", en Newmeyer, F.J. (ed.), Linguistics: The Cambridge Survey. I. Linguistic Theory: Foundations, Cambridge, Cambridge University Press, 416-429. Cit. por la trad. esp. de L.A. Santos, "Propiedades matemáticas de las gramáticas", en Panorama de la Lingüística Moderna. I. Teoria Lingüistica: Fundamentos, Madrid, Visor, 477-491. 